\title{
Editorial
}

\section{Economics and Optimizations in Wireless Communication Networks}

\author{
Lingjie Duan, ${ }^{1}$ Fei Richard Yu, ${ }^{2}$ and Lin $\mathrm{Gao}^{3}$ \\ ${ }^{1}$ Engineering Systems and Design Pillar of Singapore University of Technology and Design, Singapore \\ ${ }^{2}$ Department of Systems and Computer Engineering, Carleton University, Ottawa, ON, Canada \\ ${ }^{3}$ School of Electronic and Information Engineering, Harbin Institute of Technology, Shenzhen, Guangdong, China
}

Correspondence should be addressed to Lingjie Duan; lingjie_duan@sutd.edu.sg

Received 4 September 2016; Accepted 4 September 2016

Copyright (C) 2016 Lingjie Duan et al. This is an open access article distributed under the Creative Commons Attribution License, which permits unrestricted use, distribution, and reproduction in any medium, provided the original work is properly cited.

Wireless communication networks have evolved over time to highly complex and large-scale systems and are often operated by various decentralized network entities. Along with this evolution, traditional centralized control techniques with pure engineering concerns no longer apply due to the lack of economic design to reconcile the conflict of interest or even competition among selfish network entities. Economic incentive is also needed when emerging communication architectures require cooperation among various network entities to share limited resources (e.g., spectrum and energy) or expensive infrastructure (e.g., cellular-WiFi networks).

This special issue aims to bring together state-of-theart research contributions on the application of economic and game-theoretic models and principles to address challenges in the deployment and optimization of communication networks and services. Potential topics of this special issue include $5 \mathrm{G}$ communication systems, green communications, dynamic spectrum access, cooperative communications, cellular-WiFi networks, mobile social networks, mobile crowdsourcing, mobile cloud computing systems, mobile video caching and distribution, wireless virtualization, and game theory in communications, networking, and services. We received a total of 22 submissions, and after two rounds of rigorous review, 9 papers were accepted.

In the first paper "Fairness-Aware and Energy Efficiency Resource Allocation in Multiuser OFDM Relaying System," G. Liang et al. propose a fairness-aware resource allocation scheme for an energy-efficient communication in a cooperative orthogonal frequency division multiple (OFDM) network based on jointly optimizing the subcarrier pairing, channel-user assignment, and power allocation. In contrast to the traditional relay-based OFDM networks, in the second phase, the source is allowed to retransmit the same signal in the first phase, further improving the system capacity performance.

In the paper "Minimizing the Average Waiting Time of Unequal-Size Data Items in a Mobile Computing Environment," J.-Y. Wang studies how to minimize the waiting time for mobile computing customers, given the limited resources, and proposes near-optimal solutions to ensure service quality and computation speed.

In the paper "Coordinated Precoding for D2D Communications Underlay Uplink MIMO Cellular Networks," B. Fang et al. study the coordinated precoding problem for device-to-device (D2D) communications underlaying multiple-input multiple-output (MIMO) cellular networks. The system model considered here consists of multiple D2D user pairs attempting to share the uplink radio resources of a cellular network. Authors first formulate the coordinated precoding problem for the $\mathrm{D} 2 \mathrm{D}$ user pairs as a sum-rate maximization (SRM) problem, which is nonconvex in general, and then reformulate it as a difference convex- (DC-) type programming problem, which can be iteratively solved by employing the famous successive convex approximation (SCA) method. By introducing a price based interference management mechanism, authors further reformulate the coordinated precoding problem as a Stackelberg game. Then, a distributed precoding algorithm is developed based on the concept of Stackelberg equilibrium (SE).

In the paper "Optimal Network QoS over the Internet of Vehicles for E-Health Applications," D. Lin et al. looked at the wireless technology problem to support ubiquitous 
healthcare applications due to electromagnetic interference (caused by RF transmission) to medical sensors. It models and optimizes the QoS within the whole Internet of vehicles for E-health by allocating transmit power for each user.

In the paper "LPPS: A Distributed Cache Pushing Based K-Anonymity Location Privacy Preserving Scheme," M. Chen et al. looked at location-based services' privacy concern and proposed location privacy preserving scheme that achieves $k$-anonymity guarantee.

In the paper "Sleep Control Game for Wireless Sensor Networks," S. H. Lee et al. aim to help each wireless sensor node to control its sleep mode and reduce energy consumption without sacrificing the message latency. Game theory is applied to optimally tell how each node schedules sleep and remove unnecessary wake-up in the equilibrium.

In the paper "Resource Allocation in a Generalized Framework for Virtualized Heterogeneous Wireless Network," B. Fan et al. propose a generic framework for virtualizing heterogeneous wireless network with different radio access technologies (RATs). The authors introduce a novel virtual medium access control (VMAC) concept to converge different RAT protocols and perform inter-RAT resource allocation. To suit the proposed framework, they further propose a virtualization based resource allocation scheme and formulate the problem as a mixed combinatorial optimization, which jointly considers network access and rate allocation. First, to solve the network access problem, authors develop an adaptability ratio to model the fact that different RATs possess different adaptability to different services and adopt a Grey Relational Analysis (GRA) method to calculate the adaptability ratio. Second, authors model a Nash bargaining game where services are game players bargaining for RAT resources and derive the closed-form Nash bargaining solution (NBS).

In the paper "Cross-Layer Cooperative Power Control in Heterogeneous Multihop Networks," F. Tian et al. investigate how to perform optimal cooperative power control for the coexistence of heterogeneous multihop networks. Then, they first formulate a multiobjective optimization problem for the total power consumption of the two heterogeneous multihop networks with discretized power level and then reformulate the nonlinear constraint (relationship between power and capacity) into the linear one by piecewise linearization procedure and offer an in-depth study of cooperative power control in terms of its optimal power: the minimum power consumption with discretized power level for both heterogeneous multihop networks. Through a novel approach based on adaptive weighted sum method, they further transform the multiobjective optimization problem into a single-objective optimization problem and find the set of Pareto-optimal points iteratively.

In the paper "Novel Handover Optimization with a Coordinated Contiguous Carrier Aggregation Deployment Scenario in LTE-Advanced Systems," I. Shayea et al. deploy Coordinated Contiguous-CADS (CC-CADS) and a Novel Handover Parameters Optimization algorithm that is based on the Weight Performance Function (NHPO-WPF) for LTE-Advanced systems to enhance system performance in terms of throughput, coverage area, and connection stability and to reduce management complexity. The CC-CADS uses two contiguous Component Carriers (CCs) that have two different beam directions, and the NHPO-WPF automatically adjusts the HCPs based on the Weight Performance Function (WPF), which is evaluated as a function of the Signal-toInterference Noise Ratio (SINR), cell load, and UE's velocity. Simulation results show that the CC-CADS and the NHPOWPF algorithm provide significant enhancements in system performance over that of conventional CADSs and HPO algorithms from the literature, respectively. The integration of both solutions achieves even better performance than scenarios in which each solution is considered independently.

\section{Acknowledgments}

The guest editorial team would like to thank authors of all the papers submitted to this special issue. Given the space limitations, a number of high quality contributions could not be accommodated. The editors also wish to thank the anonymous reviewers, some of whom helped with multiple review assignments. Finally, we would like to thank the journal's Editor-in-Chief David Taniar for being very encouraging and accomodative regarding this special issue. We hope that you will enjoy reading this special issue devoted to this exciting and fast-evolving field as much as we have done.

Lingjie Duan

Fei Richard Yu

Lin Gao 

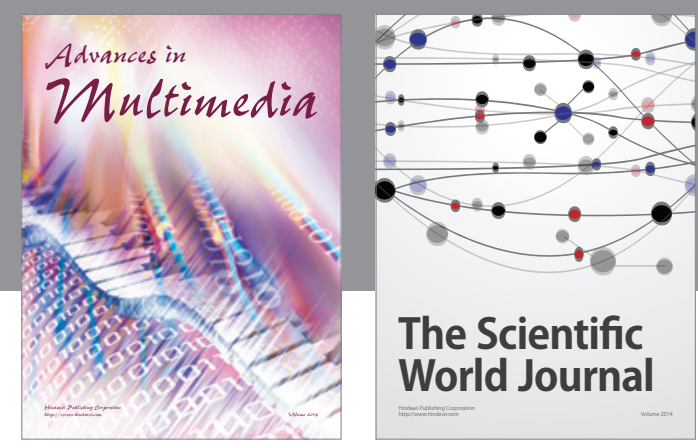

The Scientific World Journal
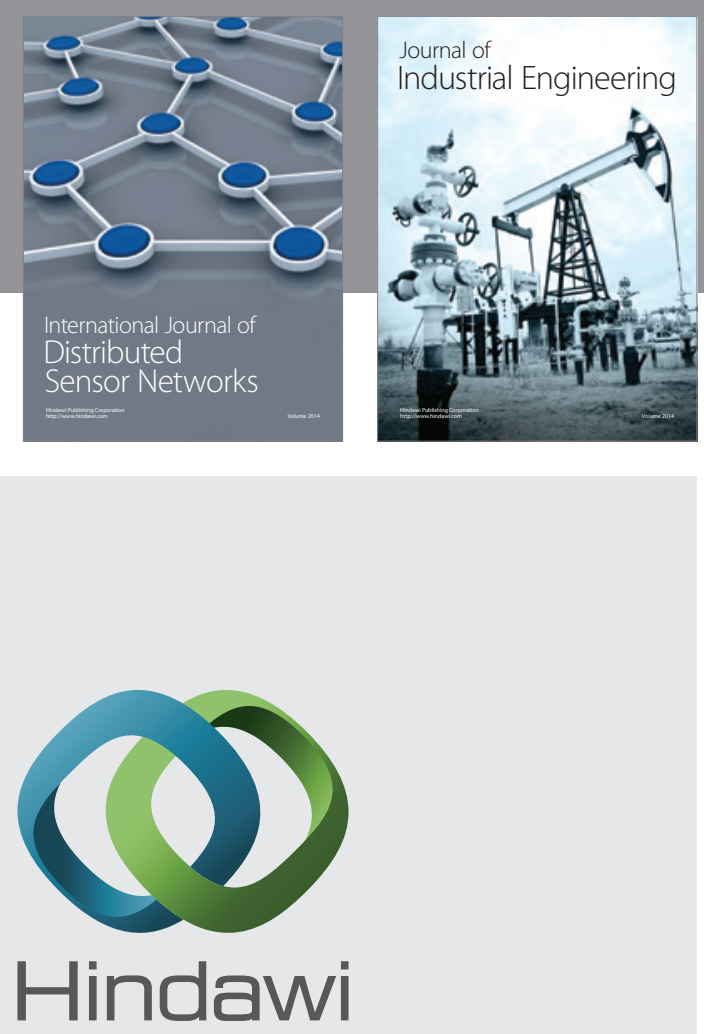

Submit your manuscripts at

http://www.hindawi.com

\section{Computer Networks} and Communications
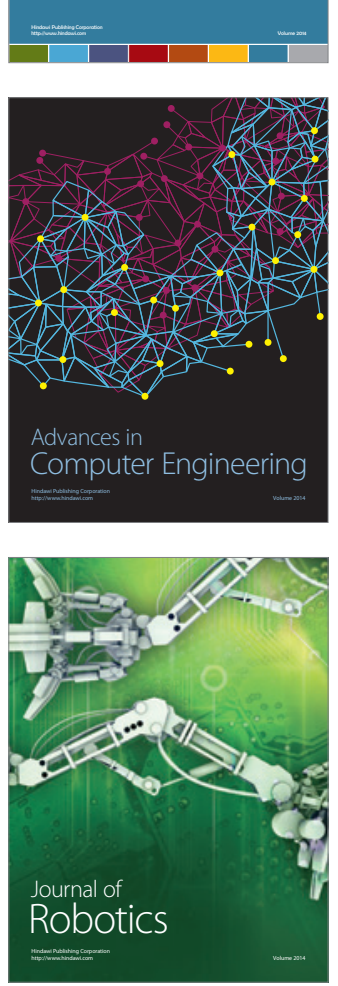
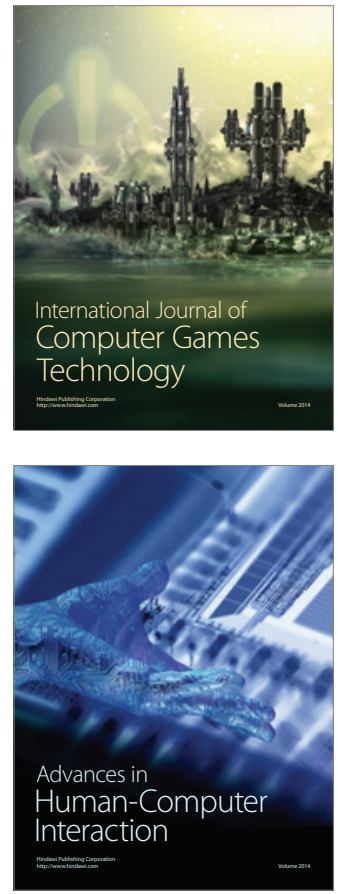
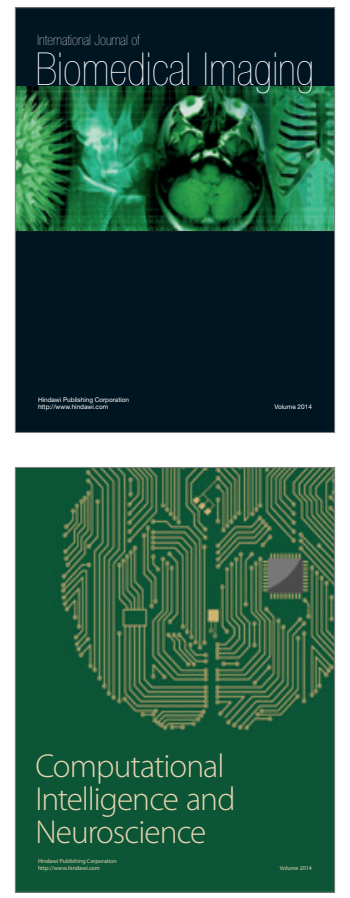
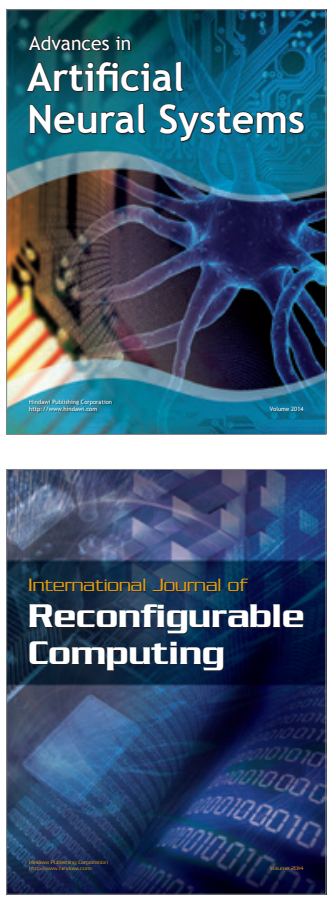
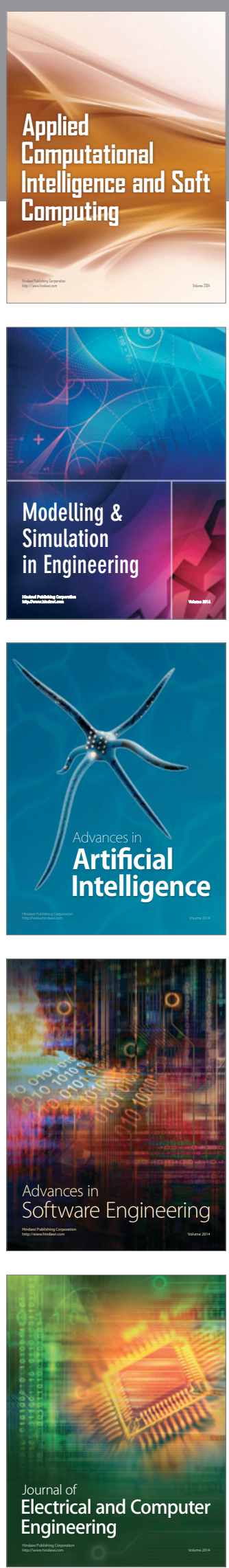